\title{
The effect of the environment on symptom dimensions in the first episode of psychosis: a multilevel study
}

\author{
F. J. Oher ${ }^{1,2}$, A. Demjaha ${ }^{3}$, D. Jackson ${ }^{4}$, C. Morgan ${ }^{5}$, P. Dazzan ${ }^{3}$, K. Morgan ${ }^{6}$, J. Boydell ${ }^{3}$, \\ G. A. Doody ${ }^{7}$, R. M. Murray ${ }^{3}$, R. P. Bentall ${ }^{8}$, P. B. Jones ${ }^{1}$ and J. B. Kirkbride ${ }^{1,9 *}$ \\ ${ }^{1}$ Department of Psychiatry, Herchel Smith Building for Brain and Mind Sciences, University of Cambridge, UK \\ ${ }^{2}$ Faculty of Medicine, Lund University, Sweden \\ ${ }^{3}$ NIHR Biomedical Research Centre, Psychosis Studies Department, Institute of Psychiatry, King's College London, UK \\ ${ }^{4} M R C$ Biostatistics Unit, University of Cambridge, UK \\ ${ }^{5}$ NIHR Biomedical Research Centre and Section of Social Psychiatry, Health Service and Population Research Department, Institute of Psychiatry, \\ King's College London, UK \\ ${ }^{6}$ Department of Psychology, University of Westminster, London, UK \\ ${ }^{7}$ Division of Psychiatry, University of Nottingham, UK \\ ${ }^{8}$ Institute of Psychology, Health and Society, University of Liverpool, UK \\ ${ }^{9}$ Division of Psychiatry, University College London, UCL
}

Background. The extent to which different symptom dimensions vary according to epidemiological factors associated with categorical definitions of first-episode psychosis (FEP) is unknown. We hypothesized that positive psychotic symptoms, including paranoid delusions and depressive symptoms, would be more prominent in more urban environments.

\begin{abstract}
Method. We collected clinical and epidemiological data on 469 people with FEP (ICD-10 F10-F33) in two centres of the Aetiology and Ethnicity in Schizophrenia and Other Psychoses (AESOP) study: Southeast London and Nottinghamshire. We used multilevel regression models to examine neighbourhood-level and between-centre differences in five symptom dimensions (reality distortion, negative symptoms, manic symptoms, depressive symptoms and disorganization) underpinning Schedules for Clinical Assessment in Neuropsychiatry (SCAN) Item Group Checklist (IGC) symptoms. Delusions of persecution and reference, along with other individual IGC symptoms, were inspected for area-level variation.
\end{abstract}

Results. Reality distortion [estimated effect size (EES) $0.15,95 \%$ confidence interval (CI) $0.06-0.24$ ] and depressive symptoms (EES 0.21, 95\% CI 0.07-0.34) were elevated in people with FEP living in more urban Southeast London but disorganized symptomatology was lower (EES $-0.06,95 \%$ CI -0.10 to -0.02 ), after controlling for confounders. Delusions of persecution were not associated with increased neighbourhood population density [adjusted odds ratio (aOR) 1.01, 95\% CI 0.83-1.23], although an effect was observed for delusions of reference (aOR 1.41, 95\% CI 1.12-1.77). Hallucinatory symptoms showed consistent elevation in more densely populated neighbourhoods (aOR 1.32, 95\% CI 1.09-1.61).

Conclusions. In people experiencing FEP, elevated levels of reality distortion and depressive symptoms were observed in more urban, densely populated neighbourhoods. No clear association was observed for paranoid delusions; hallucinations were consistently associated with increased population density. These results suggest that urban environments may affect the syndromal presentation of psychotic disorders.

Received 30 September 2013; Revised 6 December 2013; Accepted 6 December 2013; First published online 20 January 2014

Key words: Epidemiology, neighbourhood, paranoia, positive symptoms, psychosis, symptom dimensions, symptomatology, social environment, urbanicity.

\section{Introduction}

Traditional epidemiological studies of first-episode psychosis (FEP), underpinned by categorical diagnostic classifications, have identified major risk factors for both non-affective and affective psychotic disorders.

* Address for correspondence: J. B. Kirkbride Ph.D., Division of Psychiatry, 67-73 Riding House Street, London W1W 7EJ, UK. (Email: j.kirkbride@ucl.ac.uk)
Risk factors for these sets of disorders are shared [migration and ethnicity (Cantor-Graae \& Selten, 2005; Fearon et al. 2006) and childhood traumas (Laursen et al. 2007)] and unique [e.g. urban birth and upbringing (Mortensen et al. 1999), paternal age (Laursen et al. 2007), developmental delays (Jones et al. 1994) and impaired pre-morbid cognition in schizophrenia (Reichenberg et al. 2002)], suggesting that there may be both overlapping and distinct aetiological pathways to psychotic disorder. 
In the past decade psychiatric research has questioned the diagnostic utility of traditional categorical classifications (Liddle, 1987; Demjaha et al. 2009; van Os et al. 2010), suggesting that dimensional conceptualizations of symptomatic presentation may also be useful in characterizing the true psychopathology underlying psychotic illness (van Os et al. 1996; Peralta \& Cuesta, 2007; Braca et al. 2013; Russo et al. 2013). Some studies have investigated how individuallevel risk factors for categorical diagnoses map onto different psychosis symptom dimensions (Stefanis et al. 2004; Allardyce et al. 2007; Demjaha et al. 2009) but only one study has examined this in relation to environmental factors (van Os et al. 2002). In that study only positive and negative symptoms were considered and multilevel modelling was not used.

In the current study we examined the extent to which several psychosis symptom dimensions in people with FEP exhibited variance according to measures of the social environment. We hypothesized that urban living would be most strongly associated with positive psychotic symptom dimensions, and within these dimensions, specifically with paranoia. Psychological models propose that paranoid symptoms exist on a continuum with healthy functioning (Freeman et al. 2005; van Os et al. 2009), co-opt healthy processes for detecting and avoiding social threats (Moutoussis et al. 2007) and arise from pre-existing feelings of vulnerability (Freeman et al. 2002) and low self-esteem (Bentall et al. 2001). There is evidence that positive symptoms in general (Schreier et al. 2009), and paranoia in particular (Janssen et al. 2003; Bentall et al. 2012), can develop following experiences of victimization, trauma and discrimination, and in the general population it seems plausible that these kinds of experiences would be more frequently encountered in high-density, urban environments. Consistent with this hypothesis, following exposure to urban environments, paranoid individuals exhibit increased levels of anxiety, negative beliefs about others and a tendency to 'jump to conclusions' on the basis of limited data (Ellett et al. 2008). We also hypothesized that depressive symptoms would be more common in people with FEP in more urban environments because mood disorders, including unipolar depression, are elevated in urban areas (Sundquist et al. 2004; Peen et al. 2010). We had no strong a priori rationale to consider that other symptom dimensions would vary according to urban gradients.

\section{Method}

\section{Sample}

All participants who presented to services with a suspected FEP over a 2-year period (1997-1999) in the
Southeast London and Nottinghamshire centres of the Aetiology and Ethnicity in Schizophrenia and Other Psychoses (AESOP) study were eligible for inclusion (Kirkbride et al. 2006; Demjaha et al. 2009). Our Southeast London centre included the Borough of Lambeth and two-thirds of the Borough of Southwark. The Nottinghamshire catchment area included the City of Nottingham and the more suburban and rural parts of the surrounding region, including the Local Authority Districts of Broxtowe, Gedling and Rushcliffe, and the town of Hucknall. We did not include participants from a third centre (Bristol) because symptom data were not collected there. Participants had to (i) be 16-64 years old, (ii) live in the catchment areas at first contact and (iii) not have an organic basis to disorder (including possible neurological and metabolic disorders) or profound learning disability. Services bases were monitored weekly, and a leakage study minimized loss to ascertainment (Kirkbride et al. 2006). The Schedules for Clinical Assessment in Neuropsychiatry (SCAN; WHO, 1992) were administered to eligible participants as soon as possible after first contact. Participants were diagnosed using the SCAN and other available information (including case-note review and informant interview) by a clinical panel. Participants who met ICD-10 criteria for substance-induced psychosis (F10-F19), non-affective psychosis (F20-F29), bipolar affective psychosis (F30 and F31) or psychotic depression (F32 and F33) were included.

\section{Data collection}

\section{Symptom dimensions}

A.D. rated 28 major signs and symptoms of psychosis on all participants according to the SCAN Item Group Checklist (IGC), using the SCAN and all available clinical data. IGC ratings were based on both frequency and severity, and were coded as absent, moderate or severe (rated 0,1 and 2 respectively). Ambiguous ratings were resolved by consensus with P.D. Our group has previously reported the factor structure underlying these IGC symptom groups in the AESOP FEP sample, and their association with individual-level clinical and sociodemographic characteristics (Demjaha et al. 2009). In that study, a principal axis factor analysis with varimax rotation suggested that five theoretically informed and empirically driven symptom dimensions could represent symptomatology: reality distortion, negative symptoms, manic symptoms, depressive symptoms and disorganization.

In the current study we investigated the extent to which each of these symptom dimensions varied at the small-area neighbourhood level. We obtained the factor loading matrix of IGC items following 
principal axis factor analysis with varimax rotation (Supplementary Table 1, ST1) in Stata version 12 (Stata Corporation, USA), and calculated participantlevel factor scores on each symptom dimension using Stata's predict post-estimation command following factor analysis. Because scores were highly positively skewed (data available on request), we applied zero-skew transformations to each symptom dimension to minimize violating the assumption that residuals in our final multilevel regression models were normally distributed.

\section{IGC symptom clusters and items}

To test the a priori hypothesis that paranoid symptoms were specifically elevated in more urban environments, we analysed each IGC symptom item as a separate outcome in multilevel, multivariable analyses. Two IGC items on the reality distortion dimension (see ST1) were of specific interest with regard to paranoia, delusions of persecution and delusions of reference. For the reality distortion dimension we also examined whether clinical symptom clusters varied at the neighbourhood level. For each participant, IGC symptom item scores were summed to produce three ordinal clusters within the reality distortion dimension: delusions (delusions of persecution, delusions of reference, delusions of control, bizarre delusions and interpretations, miscellaneous delusions; scores ranged from 0 to 12), hallucinations (non-affective auditory hallucinations, non-specific auditory hallucinations, non-specific visual hallucinations, altered perception; scores ranged from 0 to 8 ) and other symptom items associated with reality distortion (non-specific psychotic experiences, experiences of disordered form of thoughts, depersonalization and derealization; scores ranged from 0 to 6 ).

\section{Individual-level sociodemographic and clinical variables}

We recorded sex, age, ethnicity, highest socioeconomic position and marital status (single, married, divorced/separated, widowed) at first contact for all participants using the Medical Research Council Sociodemographic Schedule (Fearon et al. 2006). Ethnicity was collapsed into seven categories: white British, white non-British, black Caribbean, black African, Indian subcontinent (Indian, Pakistani, Bangladeshi), mixed white and black Caribbean, and all other ethnicities. Highest ever socio-economic position was based on occupation, coded using the National Statistics Socio-economic Classification (NS-SEC; ONS, 2005) with six categories: professional, self-employed and intermediate occupations, supervisory roles, semi-routine occupations, routine occupations, long-term employed. We included a broad categorical ICD-10 diagnosis variable as a potential confounder, as described earlier. In addition, we recorded mode of onset [acute ( $<1$ month) versus insidious $(>1$ month)], lifetime poly-drug use prior to first contact (no use, single drug use, poly-drug use) and parental history of psychosis from the Personal and Psychiatric History Schedule, Schedule for Drug Use Assessment, Family Interview for Genetic Studies and all other data sources in the AESOP study.

\section{Area-level variables}

We included a centre-level variable to inspect whether symptom dimensions differed between our more urban [Southeast London, 2001: 95 people per hectare (pph)] and less urban (Nottinghamshire, 2001: $30 \mathrm{pph}$ ) settings. To determine whether symptom dimensions varied across smaller neighbourhoods, we geocoded participants to Office for National Statistics 2001 statistical wards (henceforth, the 'neighbourhood' or 'neighbourhood level') in which they resided at first contact with services for FEP $(n=88$ neighbourhoods; mean population: 9650). Participants of no fixed abode or who could not otherwise be geocoded were excluded. We estimated several neighbourhood-level socio-environmental exposures using data collected as close as possible to first contact, including: population density (in pph, 2001 census) (Kirkbride et al. 2007, 2012), own-group ethnic density (as a proportion of total neighbourhood population, 2001 census) (Kirkbride et al. 2007, 2012), deprivation [Index of Multiple Deprivation (IMD) scores, 2004 (Noble et al. 2004); calculated at lower super output area level and re-estimated at neighbourhood level based on a population-weighted mean (Kirkbride et al. 2007)], inequality [disparity in IMD scores across each neighbourhood at lower super output area level (Kirkbride et al. 2012); estimated using the Gini coefficient, where 0 indicated maximum equality (i.e. all lower super output areas in a neighbourhood were equally deprived) and 100 indicated maximum inequality] and 2002 local election voter turnout (percentage turnout in each neighbourhood) as a proxy for social capital (Kirkbride et al. 2007). All neighbourhood variables were $z$ standardized with a mean of zero and standard deviation (s.D.) of one.

\section{Statistical analyses}

\section{Multilevel models}

We used multilevel linear regression to inspect neighbourhood-level and centre-level differences in transformed symptom dimensions. For ordinal outcomes (symptom clusters and IGC items) we used multilevel ordinal regression. For some individual IGC items there was insufficient variation to fit ordinal 
regression models, so we collapsed the outcome into a binary variable (absent versus moderate/severe) and used multilevel logistic regression. All models were random intercept models that included a random effect to allow baseline symptomatology to vary between neighbourhoods, but assumed individual-level exposures had the same (fixed) effect across all neighbourhoods.

\section{Modelling strategy}

Treating each transformed symptom dimension separately, we first ran a null multilevel model to estimate the proportion of neighbourhood-level variance in the symptom dimension. We then added age, sex and ethnicity as a priori confounders to a model with our centre-level variable, which was retained as a fixed effect in further model building if it significantly improved model fit. Using a forward-fitting approach, we then added broad diagnostic category and other symptom dimensions to the model to see if they confounded our findings. Finally, we tested whether any neighbourhood-level socio-environmental variables improved the model fit. We reported results from the most parsimonious model for each symptom dimension. An analogous approach was adopted for ordinal IGC symptom clusters and items. Because of a substantial degree of missing data on five confounders (mode of onset, poly-drug use, parental history of psychosis, highest socio-economic position, marital status; see Table 1), we inspected their confounding effect on our results using sensitivity analyses. For each symptom dimension, we first included these five variables in the final model with missing values coded to their minimums (acute onset, no drug use, no family history, professional occupation, married) and reported the change in any neighbourhood-level effect size. We then repeated this procedure having coded missing data to their maximum values (insidious onset, polydrug use, positive family history, long-term unemployed, single).

\section{Model fit and reporting}

Model fit was assessed with Akaike's Information Criterion (AIC), with lower scores indicating a better fit. Estimated effect sizes (EES) and their 95\% confidence intervals (CIs) refer to the change in the transformed symptom dimension given a unit change in each covariate. For ordinal or binary IGC outcomes, we report adjusted odds ratios (aORs) and 95\% CIs associated with a unit change in a given covariate.

\section{Ethical standards}

All procedures contributing to this work complied with the ethical standards of the relevant national and institutional committees on human experimentation and with the Declaration of Helsinki of 1975, as revised in 2008 .

\section{Results}

Sample

Of the 535 participants who presented to services with FEP in the two centres, we had complete IGC data on $484(90.5 \%)$. Five symptom dimensions have previously been reported to underlie IGC symptoms in this sample (Demjaha et al. 2009) (ST1). We excluded a further 15 participants because (i) they lived outside the catchments at first referral $(n=3)$, (ii) they had no fixed abode $(n=11)$, or (iii) their address at first presentation could not otherwise be geocoded $(n=1)$. A larger proportion of the Nottinghamshire sample was excluded [Nottinghamshire: $22.0 \%(n=45)$ versus Southeast London: $6.4 \%(n=21), \chi^{2}=28.4,1$ degree of freedom (df), $p<0.001]$. Excluded participants did not differ on any other individual-level clinical or sociodemographic variables (data available on request).

The final sample for analysis was 469, of whom $56.7 \%$ were men (Table 1 ). The proportion of participants from an ethnic minority group $(76.7 \%$ v. $22.5 \%$, Fisher's exact $p<0.01$ ) or receiving a diagnosis of non-affective psychosis $(74.1 \%$ v. $56.9 \%$, Fisher's exact $p<0.01$ ) was greater in Southeast London than in Nottinghamshire; lifetime poly-drug use was more common in Nottinghamshire $\left(41.6 \%\right.$ v. $25.9 \%, \chi^{2}=11.2$, $1 \mathrm{df}, p<0.01)$. A smaller proportion of participants in Southeast London compared with Nottinghamshire had attained a professional occupation at first referral but were more likely to be self-employed, in intermediary occupations or long-term employed; a larger proportion of the Nottinghamshire sample were in professional, semi-routine and routine occupations (Fisher's exact $p<0.01$ ). No other statistically significant differences were observed between centres on any sociodemographic or clinical variables. Neighbourhoods in our Southeast London centre had higher median levels of population density, multiple deprivation and black and minority ethnic density, and had lower median levels of inequality and voter turnout at local elections compared with neighbourhoods in our Nottinghamshire centre (Table 2).

\section{Multilevel modelling of psychotic symptom dimensions}

Null multilevel models suggested that approximately $4.9 \%$ of variance in reality distortion $\left(\chi^{2} p=0.03\right)$ and $3.1 \%$ of variance in disorganization $\left(\chi^{2} p=0.05\right)$ could be attributed to the neighbourhood level (Supplementary Table ST2). For both symptom dimensions, 
Table 1. Participant social and clinical characteristics

\begin{tabular}{|c|c|c|c|c|}
\hline Sample characteristics at referral & $\begin{array}{l}\text { Total, } \\
N(\%)\end{array}$ & $\begin{array}{l}\text { Southeast London, } \\
N(\%)\end{array}$ & $\begin{array}{l}\text { Nottinghamshire, } \\
N(\%)\end{array}$ & Test statistics ${ }^{\mathrm{a}}$ \\
\hline Total cases & $469(100.0)$ & $309(65.9)$ & $160(34.1)$ & \\
\hline Median age, years (IQR) & $29(22-36)$ & $29(23-36)$ & $28(21-37)$ & $z=0.94, p=0.34$ \\
\hline \multicolumn{5}{|l|}{ Sex } \\
\hline Men & $266(56.7)$ & $169(54.7)$ & $97(60.6)$ & \multirow[b]{2}{*}{$\chi^{2}=1.5, p=0.22$} \\
\hline Women & $203(43.3)$ & $140(45.3)$ & $63(39.4)$ & \\
\hline \multicolumn{5}{|l|}{ Ethnicity $^{\mathrm{b}}$} \\
\hline White British & $196(41.8)$ & $72(23.3)$ & $124(77.5)$ & \multirow[b]{6}{*}{ Fisher's $p<0.01$} \\
\hline White, other ethnicities & $34(7.3)$ & $29(9.4)$ & $5(3.1)$ & \\
\hline Black Caribbean and black African & $126(26.9)$ & $176(57.0)$ & $12(7.5)$ & \\
\hline Indian subcontinent & $14(3.0)$ & $4(1.3)$ & $10(6.3)$ & \\
\hline Mixed white and black Caribbean & $10(2.1)$ & $5(1.6)$ & $5(3.1)$ & \\
\hline Other ethnicities & $27(5.8)$ & $23(7.4)$ & $4(2.5)$ & \\
\hline \multicolumn{5}{|l|}{ Highest socio-economic position } \\
\hline Professional and managerial & $44(11.3)$ & $24(9.6)$ & $20(14.5)$ & \\
\hline Intermediate and self-employed & $89(22.9)$ & $69(27.5)$ & $20(14.5)$ & \\
\hline Supervisory occupations & $29(7.5)$ & $14(5.6)$ & $15(10.9)$ & \\
\hline Semi-routine occupations & $112(28.8)$ & $68(27.1)$ & $44(31.9)$ & \\
\hline Routine occupations & $92(23.7)$ & $57(22.7)$ & $35(25.4)$ & \multirow{3}{*}{ Fisher's $p<0.01$} \\
\hline Long-term unemployed & $23(5.9)$ & $19(7.6)$ & $4(2.9)$ & \\
\hline Missing $^{c}$ & 80 [17.1] & $58[18.8]$ & $22[13.8]$ & \\
\hline \multicolumn{5}{|l|}{ Marital status ${ }^{\mathrm{b}}$} \\
\hline Single & $332(74.4)$ & $220(75.6)$ & $112(72.3)$ & \multirow{5}{*}{ Fisher's $p=0.47$} \\
\hline Married & $67(15.0)$ & $39(13.4)$ & $28(18.1)$ & \\
\hline Divorced or separated & $43(9.6)$ & N.R. & N.R. & \\
\hline Widowed & $4(0.9)$ & N.R. & N.R. & \\
\hline Missing $^{c}$ & $23[4.9]$ & $18[5.8]$ & $5[3.1]$ & \\
\hline \multicolumn{5}{|l|}{ Diagnosis $^{\mathrm{b}}$} \\
\hline Non-affective psychosis & $320(68.2)$ & $229(74.1)$ & $91(56.9)$ & \multirow[b]{3}{*}{ Fisher's $p<0.01$} \\
\hline Affective psychosis & $128(27.3)$ & N.R. & N.R. & \\
\hline Drug-induced psychosis & $21(4.5)$ & N.R. & N.R. & \\
\hline \multicolumn{5}{|l|}{ Mode of onset } \\
\hline Acute & $211(50.6)$ & $141(54.1)$ & $70(49.1)$ & \multirow{3}{*}{$\chi^{2}=1.5, p=0.70$} \\
\hline Insidious & $222(49.4)$ & $147(48.6)$ & $75(50.9)$ & \\
\hline Missing $^{c}$ & $36[7.7]$ & $21[6.8]$ & $15[9.4]$ & \\
\hline \multicolumn{5}{|l|}{ Lifetime poly-drug use } \\
\hline No use & $142(40.2)$ & $94(41.2)$ & $48(38.4)$ & \multirow{4}{*}{$\chi^{2}=11.2, p<0.01$} \\
\hline Single drug use & $100(28.3)$ & $75(32.9)$ & $25(25.0)$ & \\
\hline Poly-drug use & $111(31.4)$ & $59(25.9)$ & $52(41.6)$ & \\
\hline Missing ${ }^{c}$ & 116 [24.7] & 81 [26.2] & 35 [21.9] & \\
\hline \multicolumn{5}{|l|}{ Parental history of psychosis } \\
\hline No & $258(86.0)$ & $142(85.0)$ & $116(87.2)$ & \multirow{3}{*}{$\chi^{2}=0.3, p=0.59$} \\
\hline Yes & $42(14.0)$ & $25(15.0)$ & $17(12.8)$ & \\
\hline Missing $^{c}$ & 169 [36.0] & $142[46.0]$ & $27[16.9]$ & \\
\hline
\end{tabular}

N.R., Not reported. To preserve participant anonymity some cells (where $n<3$ ) are not reported by centre.

Values given as number (percentage) or median (interquartile range; IQR).

a $\chi^{2}$ test except for Fisher's exact test where stated when small cell values $(<5)$ encountered, or for median comparison of age by centre (Wilcoxon rank sum test). Tests compare strata of non-missing cells only.

${ }^{b}$ For the ethnicity variable, black Caribbean and black African groups have been collapsed for presentation purposes.

${ }^{\mathrm{c}}$ Total number of participants with missing data, expressed as a percentage of overall sample $(n=469)$ in square brackets. 
Table 2. Neighbourhood ward-level characteristics in the Southeast London and Nottinghamshire AESOP catchment areas

\begin{tabular}{|c|c|c|c|c|}
\hline Neighbourhood characteristics & $\begin{array}{l}\text { Total } \\
\text { median (IQR) }\end{array}$ & $\begin{array}{l}\text { Southeast London } \\
\text { median (IQR) }\end{array}$ & $\begin{array}{l}\text { Nottinghamshire } \\
\text { median (IQR) }\end{array}$ & $\begin{array}{l}\text { Wilcoxon rank } \\
\text { sum } z \text { score; } \\
p \text { value }\end{array}$ \\
\hline Population density (pph) & $42.7(24.7-84.6)$ & $94.8(81.6-119.0)$ & $30.2(12.1-42.7)$ & $z=7.3 ; p<0.01$ \\
\hline IMD score & $28.6(15.5-38.1)$ & $34.1(28.6-38.4)$ & $17.8(10.2-33.1)$ & $z=3.6 ; p<0.01$ \\
\hline IMD Inequality (\%) & $14.0(9.4-19.0)$ & $11.0(8.6-14.8)$ & $15.3(11.1-21.5)$ & $z=-3.1 ; p<0.01$ \\
\hline Local election voter turnout (\%) & $30.2(24.6-37.2)$ & $24.6(22.9-29.9)$ & $34.5(28.7-43.2)$ & $z=-4.9 ; p<0.01$ \\
\hline \multicolumn{5}{|l|}{ Own-group ethnic density (\%) } \\
\hline White British $^{\mathrm{a}}$ & $84.1(54.3-94.1)$ & $51.3(43.4-54.8)$ & $91.6(85.6-95.7)$ & $z=-7.6 ; p<0.01$ \\
\hline
\end{tabular}

AESOP, Aetiology and Ethnicity in Schizophrenia and Other Psychoses; IQR, interquartile range; pph, people per hectare; IMD, Index of Multiple Deprivation.

${ }^{\text {a }}$ For clarity of presentation, own-group ethnic density is summarized for the white British group only. In multilevel models neighbourhood own-group ethnic density is estimated for each ethnic group $(n=7)$.

univariate associations were observed with several neighbourhood-level exposures, including evidence that reality distortion was elevated in our more urban centre, Southeast London, but levels of disorganization were lower (ST2). Despite the absence of any apparent small-area neighbourhood variation in depressive symptoms, a univariate model suggested that greater symptomatology was present in the Southeast London sample. Univariate associations suggested reality distortion was also elevated in some ethnic minority groups, but otherwise there was little evidence that any symptom dimension varied by individual-level exposures.

Centre-level differences in reality distortion, depressive symptoms and disorganization remained evident after adjustment for age, sex, ethnicity, broad diagnosis and other symptom dimensions included in our final multivariate models (Fig. 1 and Table 3). Both reality distortion (EES 0.15, 95\% CI 0.06-0.24) and depressive symptoms (EES 0.21, 95\% CI 0.07-0.34) were elevated in Southeast London compared with Nottinghamshire, whereas levels of disorganization were lower (EES $0.06,95 \% \mathrm{CI}-0.10$ to -0.02$)$. These differences persisted after additional adjustment for mode of onset, polydrug use, socioeconomic position, marital status and parental history of psychosis in sensitivity analyses (Table 4). Manic and negative symptom dimensions did not vary significantly between centres after full multivariate adjustment (Fig. 1). No specific neighbourhood-level exposure improved model fit (data available on request).

\section{Multilevel modelling of IGC symptom clusters and items}

For IGC symptom clusters and items, population density provided a better fit to our models than the 'centre' variable (data available on request). Within the reality distortion dimensions, items related to hallucinations were most strongly and consistently associated with increased symptomatology among people with FEP living in more densely populated neighbourhoods (aOR associated with a 1 s.D. increase in population density: 1.32, 95\% CI 1.10-1.61; Table 5). By contrast, we did not observed any consistent evidence that delusions (aOR 1.18, 95\% CI 0.98-1.41), including delusions of persecution (aOR 1.01, 95\% CI 0.83-1.23), were associated with population density, with the exception of delusions of reference (aOR 1.41, 95\% CI 1.12-1.77). Two of the three other items associated with reality distortion (non-specific psychotic experiences, disordered form of thoughts) also showed positive associations with increased population density after adjustment for confounders (see Table 5).

For depressive symptoms, two of three IGC items on this dimension were also significantly associated with increased population density: depressive delusions and hallucinations (aOR 1.58, 95\% CI 1.00-2.48) and depressed mood (aOR 1.40, 95\% CI 1.11-1.76) (Table 5). Increased population density was associated with a lower odds of both IGC items on the disorganization dimension, although this only achieved statistical significance for 'emotional turmoil' (aOR 0.74, 95\% CI 0.60-0.92).

\section{Discussion}

\section{Principal findings}

This is the first study to have investigated possible variation in symptom dimensions according to environmental factors in a FEP sample. We observed greater levels of reality distortion and depressive symptoms, and lower levels of disorganization in our 


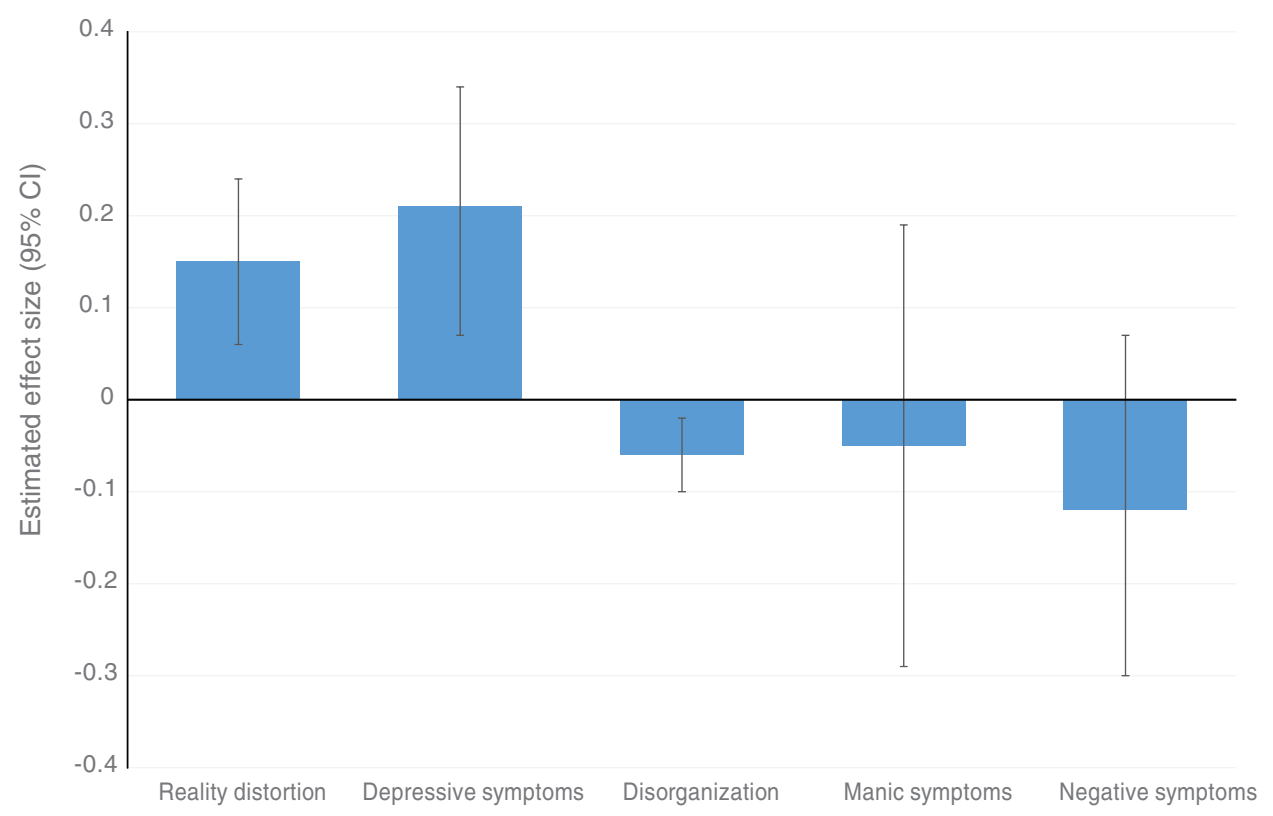

Fig. 1. Estimated effect size (EES) of change in transformed symptom dimension scores for people with first episode psychosis (FEP) in the more urban, Southeast London centre of the Aetiology and Ethnicity in Schizophrenia and Other Psychoses (AESOP) study [mean population density: 95 people per hectare (pph)], compared with their counterparts in the less urban Nottinghamshire centre (mean population density: $30 \mathrm{pph}$ ). Values of EES are adjusted for age, sex, ethnicity, broad diagnosis and any other statistically significant symptom dimensions associated with the outcome variable (see Table 3). Positive values for EES (i.e. above the baseline, Nottinghamshire sample) indicate raised symptomatology in Southeast London whereas negative values indicate reduced symptomatology in Southeast London. Statistically nonsignificant differences between the two centres in symptomatology are indicated by $95 \%$ confidence intervals (CIs) that overlap zero.

most urban setting, after adjustment for several confounders. Manic and negative symptomatology showed little area-level variation. Delusions of persecution were not elevated among people experiencing FEP in more densely populated neighbourhoods in our sample, although one other symptom relevant to paranoid thinking, delusions of reference, did show such a relationship. Within the dimension of reality distortion, we observed that increased population density was most consistently associated with changes in hallucinatory symptomatology among people with FEP in our sample.

\section{Strengths and weaknesses}

Symptom dimensions investigated in our study were based on theoretical and empirical evidence regarding underlying dimensional structure in FEP. Within the reality distortion dimension, we grouped IGC symptom clusters according to clinical knowledge; this is unlikely to have substantially biased our results. Greater endorsement of all hallucinatory IGC symptoms was associated with increased neighbourhood population density; this met conventional statistical significance on three of four items, with a strong trend in this direction for the fourth (non-specific visual hallucinations). We acknowledge that the cluster 'other symptom items associated with reality distortion' was heterogeneous. Clinically, it may be argued that symptoms of 'depersonalization and derealization' did not strictly constitute a 'positive' psychotic symptom, and this may have been borne out by the data because both 'disordered form of thoughts' and 'non-specific psychotic experiences' were significantly associated with population density, but this item was not. Given the novelty of our findings as a whole, however, and the diminished content validity inherent for any single IGC item, we suggest caution in the interpretation of results at the IGC item level.

Diagnostic data were collected by trained raters in each study centre using the SCAN, with high interrater reliability (between 0.6 and 0.8 for specific diagnoses, and 1.0 for psychotic disorder; Kirkbride et al. 2006). A single, trained rater conducted all SCAN IGC ratings to minimize differential ratings being applied between our centres. A greater proportion of participants missing IGC data $(9.5 \%$ of the original sample) were from Nottinghamshire. If they were missing not at random (MNAR), this could have led to either under- or overestimation of symptom dimensions in this centre, affecting our results. Although we could not assess this directly, we consider that any 
Table 3. Final multilevel models for symptom dimensions ${ }^{a}$ where significant area-level effects were observed

\begin{tabular}{|c|c|c|c|}
\hline & $\begin{array}{l}\text { Reality distortion } \\
\text { EES }(95 \% \mathrm{CI})\end{array}$ & $\begin{array}{l}\text { Depressive symptoms } \\
\text { EES }(95 \% \mathrm{CI})\end{array}$ & $\begin{array}{l}\text { Disorganization } \\
\text { EES }(95 \% \mathrm{CI})\end{array}$ \\
\hline \multicolumn{4}{|l|}{ Area-level fixed effects } \\
\hline Southeast London $v$. Nottinghamshire & 0.15 (0.06 to 0.24$)$ & $0.21(0.07$ to 0.34$)$ & $-0.06(-0.10$ to -0.02$)$ \\
\hline \multicolumn{4}{|l|}{ Individual-level fixed effects } \\
\hline \multicolumn{4}{|l|}{ Ethnicity } \\
\hline White British & 1 & 1 & 1 \\
\hline White, other ethnicities & $0.12(-0.03$ to 0.26$)$ & $-0.03(-0.24$ to 0.18$)$ & $0.009(-0.05$ to 0.07$)$ \\
\hline Black Caribbean & $0.03(-0.07$ to 0.13$)$ & $-0.19(-0.34 \text { to }-0.05)^{b}$ & 0.0002 (-0.04 to 0.04$)$ \\
\hline Black African & $0.10(-0.02$ to 0.23$)$ & -0.16 ( -0.33 to 0.02$)$ & $-0.02(-0.07$ to 0.04$)$ \\
\hline Indian subcontinent & $-0.01(-0.22$ to 0.21$)$ & $-0.14(-0.45$ to 0.16$)$ & $0.006(-0.07$ to 0.09$)$ \\
\hline Mixed, white and black Caribbean & $-0.14(-0.39$ to 0.10$)$ & $-0.04(-0.40$ to 0.31$)$ & $0.04(-0.05$ to 0.14$)$ \\
\hline Other ethnicities & $0.05(-0.12$ to 0.21$)$ & $-0.09(-0.35$ to 0.12$)$ & $0.005(-0.06$ to 0.07$)$ \\
\hline \multicolumn{4}{|l|}{ Diagnostic category } \\
\hline Non-affective psychotic disorder & 1 & 1 & 1 \\
\hline Affective psychotic disorder & - & - & $-0.06(-0.10 \text { to }-0.03)^{\mathrm{b}}$ \\
\hline Bipolar psychotic disorder & $-0.45(-0.58 \text { to }-0.33)^{\mathrm{b}}$ & $-0.12(-0.31$ to 0.07$)$ & - \\
\hline Depressive psychotic disorder & $-0.23(-0.33 \text { to }-0.12)^{b}$ & $0.86(0.71 \text { to } 1.02)^{\mathrm{b}}$ & - \\
\hline Substance-induced psychotic disorder & $-0.12(-0.30$ to 0.06$)$ & $-0.20(-0.46$ to 0.06$)$ & $-0.05(-0.12$ to 0.02$)$ \\
\hline \multicolumn{4}{|l|}{ Symptom dimensions } \\
\hline Manic symptoms & $0.10(0.07 \text { to } 0.13)^{\mathrm{b}}$ & $0.11(0.06 \text { to } 0.16)^{\mathrm{b}}$ & $0.02(0.006 \text { to } 0.03)^{\mathrm{b}}$ \\
\hline Negative symptoms & - & $-0.16(-0.22 \text { to }-0.09)^{b}$ & - \\
\hline Area-level random effect: ICC ( $\chi^{2} p$ value) & $0.00 \%(p=1.00)$ & $0.18 \%(p=0.47)$ & $0.69 \%(p=0.35)$ \\
\hline AIC & 471.06 & 809.87 & -433.40 \\
\hline
\end{tabular}

EES, Estimated effect size; CI, confidence interval; ICC, intraclass correlation coefficient; AIC, Akaike's Information Criterion.

${ }^{a}$ Effect sizes adjusted for age, sex and other variables in the final model, as presented. Effect sizes for age and sex not shown as no statistically significant associations with symptom dimensions were observed in the final models. All values rounded to two decimal places or one significant digit (where $>-0.01$ and $<0.01$ ).

bignificant at $p<0.05$.

Table 4. Sensitivity analysis to examine confounding effect of individual covariates containing missing data on area-level effects from final multivariate models: Southeast London versus Nottingham

\begin{tabular}{llll}
\hline & $\begin{array}{l}\text { Reality distortion } \\
\text { EES }(95 \% \mathrm{CI})\end{array}$ & $\begin{array}{l}\text { Depressive symptoms } \\
\text { EES }(95 \% \mathrm{CI})\end{array}$ & $\begin{array}{l}\text { Disorganization } \\
\text { EES (95\% CI) }\end{array}$ \\
\hline${\text { Sensitivity adjustment } 1^{\mathrm{a}}}_{\text {Sensitivity adjustment }^{\mathrm{a}}}$ & $0.16(0.06 \text { to } 0.25)^{\mathrm{b}}$ & $\begin{array}{l}0.21(0.07 \text { to } 0.35)^{\mathrm{b}} \\
0.25(0.11 \text { to } 0.39)^{\mathrm{b}}\end{array}$ & $\begin{array}{l}-0.06(-0.09 \text { to }-0.02)^{\mathrm{b}} \\
-0.06(-0.10 \text { to }-0.02)^{\mathrm{b}}\end{array}$ \\
\hline
\end{tabular}

EES, Estimated effect size; CI, confidence interval.

${ }^{\text {a }}$ Final models from Table 3 with additional control for mode of onset, parental history of psychosis, lifetime poly-drug use, socio-economic position and marital status. Sensitivity adjustment 1 coded all people with missing data on these covariates to the baseline group [no evidence of lifetime drug use, acute first-episode psychosis (FEP) onset, no parental history, professional occupation, married]. Sensitivity adjustment 2 coded all people with missing data on these covariates to the highest exposure category (poly-drug use, insidious onset, positive parental history of psychosis, long-term unemployed, single).

${ }^{\mathrm{b}} p<0.05$.

such effect would have been small, given that excluded participants did not differ from the remainder of the sample on any clinical or social characteristics. Our FEP sample was not medication naïve at IGC assessment but, using available data $(n=173)$, we found no evidence to suggest that the proportion of antipsychoticnaïve participants at baseline differed statistically significantly between centres [Southeast London 
Table 5. Association between population density and selected IGC symptom clusters (reality distortion, depressive symptoms and disorganization) and IGC symptom items

\begin{tabular}{|c|c|c|}
\hline & $\mathrm{aOR}(95 \% \mathrm{CI})^{\mathrm{a}}$ & $\begin{array}{l}\text { Multilevel regression } \\
\text { model type }\end{array}$ \\
\hline \multicolumn{3}{|l|}{ Reality distortion } \\
\hline Delusions & $1.18(0.98-1.41)$ & Ordinal logistic \\
\hline Delusions of reference & $1.41(1.12-1.77)^{\mathrm{c}}$ & Ordinal logistic \\
\hline Delusions of persecution & $1.01(0.83-1.23)$ & Ordinal logistic \\
\hline Delusions of control & $1.18(0.87-1.60)$ & Logistic \\
\hline Bizarre delusions and interpretations & $0.98(0.78-1.23)$ & Ordinal logistic \\
\hline Miscellaneous delusions & $1.26(0.99-1.60)$ & Logistic \\
\hline Hallucinations & $1.32(1.09-1.61)$ & Ordinal logistic \\
\hline Non-specific auditory hallucinations & $1.25(1.001-1.55)$ & Ordinal logistic \\
\hline Non-specific visual hallucinations & $1.39(0.97-2.00)$ & Logistic \\
\hline Non-affective auditory hallucinations & $1.33(1.04-1.71)$ & Ordinal logistic \\
\hline Altered perception & $1.65(1.15-2.36)^{\mathrm{c}}$ & Logistic \\
\hline Other symptom items associated with reality distortion ${ }^{\mathrm{e}}$ & $1.39(1.10-1.77)^{\mathrm{d}}$ & Ordinal logistic \\
\hline Non-specific psychotic experiences & $1.56(1.09-2.25)$ & Logistic \\
\hline Experiences of disordered form of thoughts & $1.39(1.04-1.87)^{\mathrm{d}}$ & Ordinal logistic \\
\hline Depersonalization and derealization & $1.13(0.80-1.60)$ & Logistic \\
\hline \multicolumn{3}{|l|}{ Depressive symptoms } \\
\hline Depressive delusions and hallucinations & $1.58(1.00-2.48)^{c, d}$ & Logistic \\
\hline Depressed mood & $1.40(1.11-1.76)$ & Ordinal logistic \\
\hline Special features of depressed mood & $1.17(0.89-1.52)$ & Logistic \\
\hline \multicolumn{3}{|l|}{ Disorganization } \\
\hline Emotional turmoil & $0.74(0.60-0.92)$ & Ordinal logistic \\
\hline Incoherent speech & $0.80(0.59-1.08)$ & Ordinal logistic \\
\hline
\end{tabular}

IGC, Item Group Checklist; aOR, adjusted odds ratio; CI, confidence interval.

${ }^{a}$ Adjusted OR for 1 standard deviation increase in population density. OR adjusted for age, sex, ethnicity and broad diagnosis. Values in bold denote statistical significance at $p<0.05$.

${ }^{\mathrm{b}}$ Default modelling was multilevel ordinal logistic regression. For some outcomes there was insufficient variation in the ordinal outcome variable to permit ordinal logistic regression models. For these analyses a binary outcome variable (absent versus moderate/severe symptomatology) was used instead and multilevel logistic modelling performed.

${ }^{\mathrm{c}}$ Additional adjustment for local election voter turnout, which significantly improved model fit.

${ }^{\mathrm{d}}$ Additional adjustment for multiple deprivation, which significantly improved model fit.

${ }^{\mathrm{e}}$ Other IGC symptom items that loaded substantively onto the reality distortion dimension (see Supplementary Table ST1).

$n=18 / 77 \quad(23.4 \%)$ versus Nottinghamshire $n=31 / 96$ $\left.(32.3 \%) ; \quad \chi^{2}=1.67, p=0.20\right] \quad$ (Pariante et al. 2005; Donoghue et al. 2012).

Our choice of confounders was guided by theoretical knowledge and the availability of data in the AESOP study. We did not control for duration of untreated psychosis (DUP) because our earlier work suggested that this did not vary at the neighbourhood level (Kirkbride et al. 2010). Categorical diagnoses met statistical significance for association with several symptom dimensions in our final models (Table 3), but did not substantially confound our main findings. Consistent with previous research (Allardyce et al. 2007; Demjaha et al. 2009; Braca et al. 2013) and theory (Peralta \& Cuesta, 2007; van Os, 2009), these data suggest that both dimensional and categorical conceptualizations of psychosis may aid aetiological research. From a subgroup analysis, statistically significant area-level differences for depressive symptoms, reality distortion and disorganization were present in both the white British and black and minority ethnic groups (Supplementary Table ST3), making residual confounding by ethnicity an unlikely explanation for these area-level differences. Residual confounding may have been present with regard to drug misuse, for which we did not have data on frequency or dosage. Given the cross-sectional study design, we were unable to determine whether drug misuse may have mediated rather than confounded associations between urbanicity and symptom dimensions. More urban environments, for example, may increase exposure to substance misuse, increasing the risk of 
some psychotic symptoms. In the UK, available data suggest a small increase in declared illicit substance misuse in urban compared with rural areas (Smith \& Flatley, 2011), although this may be confounded by several factors, including age and socio-economic position. Longitudinal studies are required to shed light on any mediating role for substance abuse between urban living and psychotic symptomatology.

Neighbourhood measures were collected as close as possible to the case ascertainment period. Population density was estimated from the 2001 census. Although this measure was estimated from data collected shortly after our case ascertainment period (2-4 years), we have no reason to believe that population density would have substantially altered across neighbourhoods during this time. It is possible that people with greater symptomatology in terms of reality distortion and depressive symptoms could have drifted into more densely populated environments as a result of social drift during the prodrome, but if this were true we would have expected to also observe greater levels of other symptom dimensions (negative, disorganized) in more urban environments; we did not.

\section{Meaning of findings}

The novelty of our findings, in combination with the limitations outlined, mean that we encourage attempts to replicate our observations in other FEP samples. In this section, we place our findings in context with existing findings to suggest further directions that may be useful for future enquiry.

Our data support the notion that environmental factors act most strongly on positive psychotic symptoms related to reality distortion. Consistent with this possibility, further inspection of the three IGC items that loaded positively on the depressive symptoms dimension indicates that 'depressive delusions and hallucinations' (aOR 1.58, 95\% CI 1.00-2.48) showed the largest effect size associated with population density (Table 5). These findings accord with current theoretical models of psychosis onset, which suggest that positive psychotic symptoms may be the result of salience dysregulation following exposure to deleterious genetic or social factors (Kapur, 2003). We suggest that densely populated environments may contribute to certain positive psychotic phenomena in two non-mutually exclusive ways.

First, growing up and living in a more densely populated, urban environment may simply increase exposure to environmental stimuli, including stimuli perceived as socially challenging, providing accumulated opportunities for dysregulation in salience and perception, perhaps through stress-related pathways (Lederbogen et al. 2011). Chronic exposure to the daily stresses of city life may, through a process such as neurochemical sensitization (Howes et al. 2004), render the brain liable to exaggerated dysfunction if additional environmental insults ensue (Pruessner et al. 2004). At the behavioural level, daily life stresses result in more psychotic-like experiences in people diagnosed with psychotic disorder and their first-degree relatives than controls (Myin-Germeys et al. 2005); this may be exaggerated in people previously exposed to major life events (Lardinois et al. 2011).

Second, the 'status syndrome' may be relevant to increased positive symptomatology seen for people with FEP in urban areas in this study. This hypothesis posits that 'how much control you have over your lifeand the opportunity you have for full social engagement and participation are crucial for health, wellbeing, and longevity' (Marmot, 2004, p. 2). Cities may expose people to several sociocultural structures, organized, for example, around social, cultural, ethnic, political or economic modalities. The competing demands and complex interactions between these societal structures in an urban environment may impede an individual's control over their immediate social environment, acting as a source of social stress or marginalization among people who are (or who perceive themselves to be) excluded from full participation and representation in their community. By contrast, more rural communities, which are typically organized into more monocultural societies with smaller social hierarchies, may allow individuals greater control over their immediate environment.

The wider empirical data in mental health are consistent with this latter possibility. Our own research suggests that urban environments marked by more social fragmentation (Allardyce et al. 2005; Kirkbride et al. 2007), including income inequality (Kirkbride et al. 2012), have elevated incidence rates of schizophrenia; this risk may be attenuated for individuals living in communities where they can draw upon others for social support (Veling et al. 2008; Kirkbride et al. 2012). In the present study, the most parsimonious interpretation of our findings suggests that people experiencing their first episode of psychosis in more densely populated urban environments exhibited more positive and depressive psychotic symptoms and less disorganization. Our data are consistent with the possibility that aspects of the environment can alter the syndromal presentation of FEP, particularly with regard to positive psychotic phenomenology.

\section{Supplementary material}

For supplementary material accompanying this paper visit http://dx.doi.org/10.1017/S0033291713003188. 


\section{Acknowledgements}

J. B. Kirkbride is a Sir Henry Wellcome Research Fellowship and is grateful to the Wellcome Trust for their support. P. B. Jones directs the National Institute for Health Research (NIHR) Collaboration for Leadership in Applied Health Research and Care (CLAHRC) for Cambridgeshire and Peterborough.

This work was supported by the Wellcome Trust (grant no. WT085540 to JBK), the National Institute for Health Research (grant RP-PG-0606-1335 to P.B.J.), the UK Medical Research Council (grant no. G9628058) and the Stanley Medical Research Institute.

\section{Declaration of Interest}

Professor R. M. Murray is an editor for Psychological Medicine and has received honoraria from Janssen, Astra-Zeneca, Lilly, BMS and Roche.

\section{References}

Allardyce J, Gilmour H, Atkinson J, Rapson T, Bishop J, McCreadie RG (2005). Social fragmentation, deprivation and urbanicity: relation to first-admission rates for psychoses. British Journal of Psychiatry 187, 401-406.

Allardyce J, McCreadie R, Morrison G, van Os J (2007). Do symptom dimensions or categorical diagnoses best discriminate between known risk factors for psychosis? Social Psychiatry and Psychiatric Epidemiology 42, 429-437.

Bentall RP, Corcoran R, Howard R, Blackwood N, Kinderman P (2001). Persecutory delusions: a review and theoretical integration. Clinical Psychology Review 21, 1143-1192.

Bentall RP, Wickham S, Shevlin M, Varese F (2012). Do specific early-life adversities lead to specific symptoms of psychosis? A study from the 2007 Adult Psychiatric Morbidity Survey. Schizophrenia Bulletin. Published online: 10 April 2012. doi: 10.1093/schbul/sbs049.

Braca M, Berardi D, Mencacci E, Murri MB, Mimmi S, Allegri F, Mazzi F, Menchetti M, Tarricone I (2013). Understanding psychopathology in migrants: a mixed categorical-dimensional approach. International Journal of Social Psychiatry. Published online: 2 June 2013. doi: 10.1177/0020764013484237.

Cantor-Graae E, Selten J-P (2005). Schizophrenia and migration: a meta-analysis and review. American Journal of Psychiatry 162, 12-24.

Demjaha A, Morgan K, Morgan C, Landau S, Dean K, Reichenberg A, Sham P, Fearon P, Hutchinson G, Jones PB, Murray RM, Dazzan P (2009). Combining dimensional and categorical representation of psychosis: the way forward for DSM-V and ICD-11? Psychological Medicine 39, 1943-1955.

Donoghue K, Mazzoncini R, Hart J, Zanelli J, Morgan C, Dazzan P, Morgan KD, Murray RM, Jones PB,
Doody GA (2012). The differential effect of illicit drug use on cognitive function in first-episode psychosis and healthy controls. Acta Psychiatrica Scandinavica 125, 400-411.

Ellett L, Freeman D, Garety PA (2008). The psychological effect of an urban environment on individuals with persecutory delusions: the Camberwell walk study. Schizophrenia Research 99, 77-84.

Fearon P, Kirkbride JB, Morgan C, Dazzan P, Morgan K, Lloyd T, Hutchinson G, Tarrant J, Fung WL, Holloway J, Mallett R, Harrison G, Leff J, Jones PB, Murray RM (2006). Incidence of schizophrenia and other psychoses in ethnic minority groups: results from the MRC AESOP Study. Psychological Medicine 36, 1541-1550.

Freeman D, Garety PA, Bebbington PE, Smith B, Rollinson R, Fowler D, Kuipers E, Ray K, Dunn G (2005). Psychological investigation of the structure of paranoia in a non-clinical population. British Journal of Psychiatry $\mathbf{1 8 6}$ 427-435.

Freeman D, Garety PA, Kuipers E, Fowler D, Bebbington PE (2002). A cognitive model of persecutory delusions. British Journal of Clinical Psychology 41, 331-347.

Howes OD, McDonald C, Cannon M, Arseneault L, Boydell J, Murray RM (2004). Pathways to schizophrenia: the impact of environmental factors. International Journal of Neuropsychopharmacology 7 (Suppl. 1), S7-S13.

Janssen I, Hanssen M, Bak M, Bijl RV, de Graaf R, Vollebergh W, McKenzie K, van Os J (2003). Discrimination and delusional ideation. British Journal of Psychiatry 182, 71-76.

Jones P, Rodgers B, Murray R, Marmot M (1994). Child development risk factors for adult schizophrenia in the British 1946 birth cohort. Lancet 344, 1398-1402.

Kapur S (2003). Psychosis as a state of aberrant salience: a framework linking biology, phenomenology, and pharmacology in schizophrenia. American Journal of Psychiatry 160, 13-23.

Kirkbride JB, Barker D, Cowden F, Stamps R, Yang M, Jones PB, Coid JW (2008). Psychoses, ethnicity and socio-economic status. British Journal of Psychiatry 193, $18-24$.

Kirkbride JB, Fearon P, Morgan C, Dazzan P, Morgan K, Tarrant J, Lloyd T, Holloway J, Hutchinson G, Leff JP, Mallett RM, Harrison GL, Murray RM, Jones PB (2006). Heterogeneity in incidence rates of schizophrenia and other psychotic syndromes: findings from the 3-center AESOP study. Archives of General Psychiatry 63, 250-258.

Kirkbride JB, Jones PB, Ullrich S, Coid JW (2012). Social deprivation, inequality, and the neighborhood-level incidence of psychotic syndromes in East London. Schizophrenia Bulletin. Published online: 12 December 2012. doi:10.1093/schbul/sbs151.

Kirkbride JB, Lunn DJ, Morgan C, Lappin JM, Dazzan P, Morgan K, Fearon P, Murray RM, Jones PB (2010). Examining evidence for neighbourhood variation in the duration of untreated psychosis. Health and Place 16, 219-225.

Kirkbride JB, Morgan C, Fearon P, Dazzan P, Murray RM, Jones PB (2007). Neighbourhood-level effects on psychoses: 
re-examining the role of context. Psychological Medicine 37, 1413-1425.

Lardinois M, Lataster T, Mengelers R, van Os J, Myin-Germeys I (2011). Childhood trauma and increased stress sensitivity in psychosis. Acta Psychiatrica Scandinavica 123, 28-35.

Laursen TM, Munk-Olsen T, Nordentoft M, Bo Mortensen P (2007). A comparison of selected risk factors for unipolar depressive disorder, bipolar affective disorder, schizoaffective disorder, and schizophrenia from a Danish population-based cohort. Journal of Clinical Psychiatry 68, 1673-1681.

Lederbogen F, Kirsch P, Haddad L, Streit F, Tost H, Schuch P, Wust S, Pruessner JC, Rietschel M, Deuschle M, Meyer-Lindenberg A (2011). City living and urban upbringing affect neural social stress processing in humans. Nature 474, 498-501.

Liddle PF (1987). The symptoms of chronic schizophrenia. A re-examination of the positive-negative dichotomy. British Journal of Psychiatry 151, 145-151.

Marmot MG (2004). The Status Syndrome: How Social Standing Affects Our Health and Longevity. Bloomsbury Publishing Plc: London.

Mortensen PB, Pedersen CB, Westergaard T, Wohlfahrt J, Ewald H, Mors O, Andersen PK, Melbye M (1999). Effects of family history and place and season of birth on the risk of schizophrenia. New England Journal of Medicine 340, 603-608.

Moutoussis M, Williams J, Dayan P, Bentall RP (2007). Persecutory delusions and the conditioned avoidance paradigm: towards an integration of the psychology and biology of paranoia. Cognitive Neuropsychiatry 12, 495-510.

Myin-Germeys I, Delespaul P, van Os J (2005). Behavioural sensitization to daily life stress in psychosis. Psychological Medicine 35, 733-741.

Noble M, Wright G, Dibben C, Smith G, McLennan D, Anttila C, Barnes H, Mokhtar C, Noble S, Avenell D, Gardner J, Covizzi I, Lloyd M (2004). Indices of Deprivation 2004. Office of the Deputy Prime Minister (ODPM): London.

ONS (2005). The National Statistics Socio-economic Classification User Manual. Office for National Statistics: London.

Pariante CM, Dazzan P, Danese A, Morgan KD, Brudaglio F, Morgan C, Fearon P, Orr K, Hutchinson G, Pantelis C, Velakoulis D, Jones PB, Leff J, Murray RM (2005). Increased pituitary volume in antipsychotic-free and antipsychotic-treated patients of the AEsop first-onset psychosis study. Neuropsychopharmacology 30, 1923-1931.

Peen J, Schoevers RA, Beekman AT, Dekker J (2010). The current status of urban-rural differences in psychiatric disorders. Acta Psychiatrica Scandinavica 121, 84-93.

Peralta V, Cuesta MJ (2007). A dimensional and categorical architecture for the classification of psychotic disorders. World Psychiatry 6, 100-101.

Pruessner JC, Champagne F, Meaney MJ, Dagher A (2004). Dopamine release in response to a psychological stress in humans and its relationship to early life maternal care: a positron emission tomography study using [11C]raclopride. Journal of Neuroscience 24, 2825-2831.

Reichenberg A, Weiser M, Rabinowitz J, Caspi A, Schmeidler J, Mark M, Kaplan Z, Davidson M (2002). A population-based cohort study of premorbid intellectual, language, and behavioral functioning in patients with schizophrenia, schizoaffective disorder, and nonpsychotic bipolar disorder. American Journal of Psychiatry 159, 2027-2035.

Russo M, Levine SZ, Demjaha A, Di Forti M, Bonaccorso S, Fearon P, Dazzan P, Pariante CM, David AS, Morgan C, Murray RM, Reichenberg A (2013). Association between symptom dimensions and categorical diagnoses of psychosis: a cross-sectional and longitudinal investigation. Schizophrenia Bulletin. Published online: 9 May 2013. doi:10.1093/schbul/sbt055.

Schreier A, Wolke D, Thomas K, Horwood J, Hollis C, Gunnell D, Lewis G, Thompson A, Zammit S, Duffy L, Salvi G, Harrison G (2009). Prospective study of peer victimization in childhood and psychotic symptoms in a nonclinical population at age 12 years. Archives of General Psychiatry 66, 527-536.

Smith K, Flatley J (2011). Drug Misuse Declared: Findings from the 2010/11 British Crime Survey. Office for National Statistics: London.

Stefanis NC, Delespaul P, Henquet C, Bakoula C, Stefanis CN, van Os J (2004). Early adolescent cannabis exposure and positive and negative dimensions of psychosis. Addiction 99, 1333-1341.

Sundquist K, Frank G, Sundquist J (2004). Urbanisation and incidence of psychosis and depression: follow-up study of 4.4 million women and men in Sweden. British Journal of Psychiatry 184, 293-298.

van Os J (2009). A salience dysregulation syndrome. British Journal of Psychiatry 194, 101-103.

van Os J, Fahy TA, Jones P, Harvey I, Sham P, Lewis S, Bebbington P, Toone B, Williams M, Murray R (1996). Psychopathological syndromes in the functional psychoses: associations with course and outcome. Psychological Medicine 26, 161-176.

van Os J, Hanssen M, de Graaf R, Vollebergh W (2002). Does the urban environment independently increase the risk for both negative and positive features of psychosis? Social Psychiatry and Psychiatric Epidemiology 37, 460-464.

van Os J, Kenis G, Rutten BPF (2010). The environment and schizophrenia. Nature 468, 203-212.

van Os J, Linscott RJ, Myin-Germeys I, Delespaul P, Krabbendam L (2009). A systematic review and meta-analysis of the psychosis continuum: evidence for a psychosis proneness-persistence-impairment model of psychotic disorder. Psychological Medicine 39, 179-195.

Veling W, Susser E, van Os J, Mackenbach JP, Selten J-P, Hoek HW (2008). Ethnic density of neighborhoods and incidence of psychotic disorders among immigrants. American Journal of Psychiatry 165, 66-73.

WHO (1992). Schedule for Clinical Assessment in Neuropsychiatry. World Health Organization: Geneva. 